\section{L'Actualité économique}

L'ACTUALITÉ

ÉCONOMIQUE

\section{François Étienne Cugnet et les Forges de Saint-Maurice Un type d'entrepreneur et d'entreprise en Nouvelle-France}

\section{Cameron Nish}

Volume 42, numéro 4, janvier-mars 1967

URI : https://id.erudit.org/iderudit/1003442ar

DOI : https://doi.org/10.7202/1003442ar

Aller au sommaire du numéro

Éditeur(s)

HEC Montréa

ISSN

0001-771X (imprimé)

1710-3991 (numérique)

Découvrir la revue

Citer ce document

Nish, C. (1967). François Étienne Cugnet et les Forges de Saint-Maurice : un type d'entrepreneur et d'entreprise en Nouvelle-France. L'Actualité économique, 42(4), 884-900. https://doi.org/10.7202/1003442ar d'utilisation que vous pouvez consulter en ligne. 


\title{
François Etienne Cugnet et les Forges de Saint-Maurice
}

\author{
Un type d'entrepreneur et d'entreprise \\ en Nouvelle-France
}

La présente étude sur François-Etienne Cugnet et les Forges de Saint-Maurice a commencé par la publication de documents sur l'entreprise elle-même. Ce point de départ nous a permis de voir quels étaient les moyens de financement de l'entreprise en Nouvelle. France, et, ensuite, toujours à l'aide de documents, d'illustrer le type d'organisation sociale, économique et politique de la société coloniale ${ }^{1}$.

François-Étienne Cugnet était à la fois homme de commerce, membre et premier conseiller au Conseil supérieur, directeur du Domaine d'Occident et ami intime de l'intendant Gilles Hocquart. Il était le «parfait négociant » en Nouvelle-France. Les quelques pages qui suivent en guise de conclusion aux documents pré sentés dans les livraisons antérieures, ont pour but de montrer à la fois la complexité de l'entreprise en Nouvelle-France, c'est-à-dire les liens économiques, politiques et sociaux qui étaient en jeu, et les problèmes d'épanouissement dans un pays que l'on qualifierait aujourd'hui de sous'développé.

François-Etienne Cugnet naquit en France, en 1688 \%. Son en trée sur la scène canadienne se fit en qualité de représentant de

x. Voir l'appendice à la fin de ce travail pour une liste des documents reproduits dans L'Actualité Économique.

2. Les colonies anglaises, le Massachusetts et le Maryland, par exemple, eurent des problèmes semblables. Voir E.N. Hartley, Ironworks. on the Saugus. Norman : University of Oklahoma Press, 1957 et Keach Johnson, \& The Genesis of the Baltimore Iron Works, Journal of Southernhistory, vol. XIX, May 1952, pp. 157.179, et la thèse de doctorat du même auteur, The Establishment of the Baltimore Company. a Case-Study of the American Iron Industry in the Eighteenth Century, State Uni. versity of Iowa, 1949 . 
Lambert, le général des fermes du Domaine d'Occident ${ }^{3}$. L'État français avait fourni à lui et à sa femme, ainsi qu'à un commis et un valet, un passage sur un navire du roi ${ }^{4}$. Il était alors âgé de trente et un ans et n'avait pas d'enfant ${ }^{5}$. Son premier fils, François-Joseph, naquit environ neuf mois après son arrivée dans la colonie, soit le 27 juin $1720^{\circ}$. Jusqu'à ce qu'il bâtit sa propre maison, il loua un local rue Saint-Pierre, à Québec, appartenant à la succession de feu Jean-François Martin de Lino ${ }^{7}$. L'intendant Hocquart nous dit, qu'en 1733, Cugnet achevait de bâtir sa maison dans la basse ville de Québec, toujours située rue Saint-Pierre ${ }^{8}$. C'était, sans doute, une maison de luxe étant donné que le toit était de fer blanc $^{\circ}$. Cugnet, dans le bilan qu'il a fait en 1742 , disait qu'elle lui avait coûté 50,000 livres ${ }^{10}$. Il était aussi propriétaire de deux autres emplacements, et d'une seigneurie sur la rivière du Sault-de-la-Chaudière, sur laquelle il avait un domaine et deux habitants. Ces propriétés étaient évaluées, par Cugnet, à treize mille livres ${ }^{11}$.

Dès 1721, le gouverneur Vaudreuil et l'intendant Bégon proposèrent Cugnet pour un poste au Conseil supérieur de Québec ${ }^{12}$. Le Conseil de la Marine refusa cette demande disant qu'il y aurait peut-être conflit d'intérêt entre sa position comme directeur du Domaine d'Occident et son poste au Conseil supérieur ${ }^{13}$. Un peu

3. Adélard Gascon, L'Oeuvre de François-Joseph Cugnet. Étude historique. Ottawa : Université d'Ottawa, thèse de maitrise, x94I, p. 2. (François-Joseph était le fils de François-Étienne.)

4. \& François-Ettienne Cugnet au nom et comme fondé de procuration de Me Ayma (sic) Lambert ajudicaire general des fermes unies de France et du Domaine d'Occident... \, A.P.C., C 11 A, 40, pp. 305.307. p. 506 .

5. « Conseil de la Marine à Beauharnois (IIIntendant), A.P.C., B, vol. 42, i 719 ,

6. Marine Leland, «François-Joseph Cugnet $(1720 \cdot 1789) \gg$, Revue de l'Univer. site Laval vol. XVI, no r, $196 \mathrm{r}, \mathrm{p} .7$.

7. R.P.L. Le Jeune, Dictionnaire général du Canada, vol. r, Ottawa : Université d'Ottawa, 1930, p. 453.

8. 24 janvier 1722 - Ordonnance qui condamne François-Étienne Cugnet, directeur de la Ferme du Domaine d'Occident, à réparer incessamment la maison qu'il avait louée, rue Saint-Pierre, à Québec, de la succession de feu Jean-François Martin de Lino, procureur du roi en la prévôté de Québec $\gg, P . G$. Roy, Inventaires des ordonnances des intendants de la Nouvelle-France, vol. I, Beauceville: l'Éclaireur, 1919, p. 216.

9. \& Beauharnois et Hocquart au Ministre, Québec, 9 8'bre, r 733 \$, A.P.C., C " A, 59, p. 149 .

ro. lbid.

11. \& Etat de mes Effets actyfs et Passifs, Québec, 21, 22, 26, 27 aoust 1742 , A.P.C., C II A, I14.1, f 215. (Dorénavant cité « État»).

12. \& Etat $\gg, f 215$.

13. P.-G. Roy, La famille Guillimin \$. Bulletin des recherches historiques, I9r7, p. 98 . 


\section{L'ACTUALITE ECONOMIQUE}

plus tard, en 1727, le gouverneur Beauharnois le proposa comme greffier du Conseil ${ }^{14}$. L'intendant Dupuy fit de même; et alla un peu plus loin en le recommandant pour le poste de procureur général, s'il n'y avait pas de conflit d'intérêt ${ }^{15}$. Le Conseil de la Marine ne suivit aucune de ces recommandations, mais en 1730 , on changea d'avis et Cugnet fut nommé membre, c'est-à-dire conseiller au Conseil supérieur ${ }^{16}$. Les observations sur la vie et les mœurs de Cugnet sont faites par Mathieu Martin de Lino, JeanBaptiste Couillard, sieur de Lespinay et Jean Eustache Lanoullier de Boisclerc, le Grand Voyer de la Nouvelle-France ${ }^{17}$. Cugnet, dans un document qu'il a soumis sur sa vie et ses mours, se dit «avocat au Parlement de Paris ${ }^{18}$. Le nouveau conseiller, juriste en bonne forme, écrit la même année au ministre de la Marine, Maurepas. Il porte à l'attention de ce dernier que sa nomination au Conseil et ses fonctions sont décrites comme "droits y appar. tenant... », plutôt que selon la formule habituelle «droits dont jouissent les conseillers des autres conseils supérieurs du royaume ». Il réclame ces derniers en disant qu'il ne veut pas que sa nomination, au terme noté, soit un précédent ${ }^{10}$. Deux ans plus tard, en 1732 , on propose Cugnet comme étant celui qui peut le mieux remplir la fonction de premier conseiller ${ }^{20}$. Il est reçu à ce poste le 18 juillet $1733^{21}$. En même temps que Cugnet est membre du Conseil supérieur et premier conseiller, il conserve son poste comme

p. $34 \mathrm{I}$

14. « Le Conseil de la Marine à Bégon, Paris; 14 juin r721 », A.P.C., B, 44-1,

15. « Beauharnois au Ministre, Québec I 8 octobre 1727 \$, A.P.C̣., C I I A, 49, p. 156.

16. Dupuy au Ministre, Québec, 20 octobre 1727 \, A.P.C., C 1 I A, Diverses Demandes, $120 \cdot 1$, p. I 5 .

17. « Ministre à Verthamont, Marly, 20 janvier I728»; \&Ministre à Vertha. mont, Versailles, 28 février I728 \$, A.P.C., B, 51, pp. ro-1 I ; 54.1, pp. 22. Voir aussi \& Président du Conseil de la Marine à MM. Beauharnois et Hocquart, Versailles, 28 mars I730 $\gg$, dans P.-G. Roy, Inventaire des papiers de Léry conservés aux archives de la province de Québec, vol. r, Beauceville : I'Eclaireur, 1939, p. 227.

r 8. Information faitte par nous Mathieu Martin Delino... Jean Baptiste Couillar, sr de Lespinay... Jean Eustache Lanoullier de Boisclerc, grand Voyer... (I730) $\gg$, A.Q., Collection de pièces judiciaires, notariales, etc., etc., conservées aux archives judiciaires de 2uébec, no 2067. (Dorénavant Collection).

19. \& Information sur la vie et les mœurs de François Étienne Cugnet, $x_{3}$ février r $730 \gg$. Collection, no 2067.

20. Cugnet au Ministre, Québec, 18 octobre I730 \$, A.P.C., C I I A, 53, p. 354 .

21. Beauharnois au Ministre, Québec, I 3 octobre 1732 \, A.P.C., C I 1 A, 57 , p. 324 . 
directeur du Domaine d'Occident ${ }^{22}$. Le revenu de Cugnet provenant de ses postes administratifs, soit comme directeur du Domaine et premier conseiller, était de 3,900 livres. Ceci représente six fois et demie le salaire moyen d'un artisan, celui-ci étant de six cents livres par année ${ }^{23}$.

Cugnet ne négligea pas ses affaires commerciales. Avec un nommé Gatineau, il entreprit, en 1730, la domestication des boufs d'Illinois. Ils demandèrent une subvention au ministre, et reçurent la promesse d'être payés 1,000 livres pour chaque bœuf d'Illinois qu'ils pourraient amener dans la colonie ${ }^{24}$. Malheureusement, l'expérience fut ratée. Gatineau, l'associé de Cugnet, parvint à envoyer quelques « veaux de lait», mais ceux'ci ne survécurent que de dix à quinze jours. Le ministre Maurepas était convaincu que l'expérience de Cugnet démontrait qu'on ne pouvait rien espérer d'un tel projet ${ }^{25}$.

Une autre entreprise de Cugnet, qui a été une petite réussite, fut celle du tabac du Canada. En 1736, par exemple, il envoya 600 livres de tabac canadien en France. Les tabacs cultivés à l'île d'Orléans et dans les environs de Montréal étaient meilleurs que ceux qui étaient cultivés à Trois-Rivières. Bien que la culture et l'exportation du tabac canadien aient continué jusqu'à la fin du régime français, ce ne fut pas un très grand succès étant donné que les Français s'habituaient mal au goût du tabac colonial ${ }^{26}$.

22. «Ministre à Cugnet, Versailles, 14 avril I $733 \$$; « Ministre à Beauharnois et Hocquart, Versailles, I4 avril I733», A.P.C., B, 59.1, pp. I64; I 42.

Voir aussi \& Requête de Cugnet d’être reçu comme premier Conseillers, 18 avril I $733 \gg$, Collection, no 2067, et « Arrêt qui reçoit François Etienne Cugnet en la charge de premier conseiller au Conseil Supérieur a la place du sieur de Lino décédé... I 8 juillet I733 $\gg$, P.G. Roy, Inventaire des jugements et délibérations du Conseil supérieur de la Nouvelle-Prance, vol. 3, Beauceville : L'Éclajreur, x934, p. 5.

23. « Ministre à Hocquart, Versailles, 12 may 1733 \$, A.P.C., B, 59-1, pp. 305. 306. Sur le Domaine d'Occident, voir Cameron Nish, « Documents relatifs à l'histoire economique du régime français, I : Les budgets de la Nouvelle. France $\gg$, L'Actualité Économique, vol. 40, no 3, octobre-décembre I964, pp. 633.635 .

24. « Mémoire concernant le régie, 4 juin I $749 \gg ; ~ « D e p e n s e s$ de Canada, $1733 \gg$, A.P.C., $\mathrm{D} 2 \mathrm{D}$, carton $\mathrm{I}$; et $\mathrm{P} \mathrm{I}, 3 \mathrm{I}$, ff 58.59 . Les statistiques sur les salaires des artisans sont tirées d'une étude intitulée La bourgeoisie canadienne, 1729-1748. Cet ouvrage sera publié à l'automne de 1967 dans la série « Histoire économique et sociale du Canada Français $\gg$ des Éditions Fides.

25. \&eauharnois et Hocquart au Ministre, Québec, r5 octobre r730»; \& Cugnet au Ministre, Québec, 18 octobre I 730 ; \& Convention entre Gatineau et Beauharnois et Hocquart, 2 I juin I73I $\gg$; $\&$ Ministre à Beauharnoie et Hocquart, Versailles, 8 avril 1732 \$, A.P.C., C I I A, 52, pp. $90.94 ; 53$, pp. 356.357 ; 54, pp. 221.222 ; B, $57 \cdot 1$, p. 8 r.

26. « Beauharnois et Hocquart au Ministre, Québec, 12 octobre I732 > \& Ministre à Beauharnois et Hocquart, Versailles, 25 mars 1733 \$, A.P.C., C 11 A, 57, pp. 99. 100 ; $\mathrm{B}, 59 \cdot \mathrm{I}, \mathrm{pp} .85 .86$. 
En 1736, Cugnet se voyait accorder le monopole de la traite des postes de Tadoussac ${ }^{27}$. Cette entreprise, que nous allons examiner davantage plus loin, est un autre exemple d'un commerce qui conduit toujours à une perte mais que tout le monde veut détenir. Maurepas, le ministre de la Marine, ne pouvait pas comprendre que le commerce de Tadoussac pût rapporter un profit de 20,495 livres et 18 sols en 1733, quand la Régie de Tadoussac était une régie d'État, et qu'en 1734, alors qu'il était question d'accorder un bail pour la traite, le profit n'était plus que de 1,535 livres 17 sols et 4 deniers, et en 1735, plus bas encore soit, de 456 livres et 2 deniers. Une fois le bail accordé à Cugnet au prix de 4,500 livres par année, le profit pour l'année 1736, montera en flèche à 6,000 livres ${ }^{28}$.

L'idée d'établir des forges en Nouvelle-France date de bien avant le XVIII siècle. En 1666, le sieur de la Tesserie avait fait un voyage dans les terres au nord de la baie Saint-Paul, dans le but d'enquêter sur les mines de fer ${ }^{20}$.

Entre 1685 et 1689, les sieurs Pourial, Boula, et Hameau suggé' rèrent l'établissement de forges au Canada. Ni la colonie, ni la métropole n'étaient prêtes à accepter ces propositions. Le seul résultat fut un procès intenté par Pierre Hameau, le maître des forges qui avait visité la Nouvelle-France, contre un de ses associés, Pourial, qu'il décrit comme «avocat de profession plus propre à conduire un procès qu'il forge... $\gg^{30}$.

Vaudreuil et Bégon, en 1717, reviennent à la charge. Ils notent qu'il y a des mines de fer à Trois-Rivières. Mais bien que le document ait été apporté au régent, le duc d'Orléans, on y a noté en date du 16 janvier 1717 : « on ne juge pas à propos de travailler

27. « Ministre à Beauharnois et Hocquart, Versailles, I 7 avril I $736 »$; «Beauhar nois et Hocquart au Ministre, Québec, 5 octobre 1737》; \& Ministre à Fafon, Versailles, 3 février I $740 \gg$, A.P.C., B, 64.3, pp. 528.529; C II A, 67, p. 20 ; B, $71 \cdot 1$, pp. $22 \cdot 23$.

28. Ministre à Beauharnois et Hocquart, Versailles, 23 avril 1738 \, A.P.C., B, 66, pp. $128 \cdot 132$.

29. \& Voyage du Sr de la Tesserie pour la mine de fer à 4 lieues dans les terres Nord de la Baye St. Paul, is aoust I666», A.P.C., C II A, III/2, pp. 201/216.

3o. \& Propositions des Srs Pourial, Boula et Hameau pour l'etablissement des forges en Canada, s.d., (circa $1685 \cdot 1686$ ) \; « Mémoire du Maistre de Forges qui a esté envoyé en Canada pour visiter les mines et y faire des Establissement, (joint à la lettre de Denonville du 6 novembre r687) \$; «P. Hameau a Monseigneur de Lagny (Directeur General du Commerce de France), (circa I688) 》, A.P.C., C I I A, Ir I/2, pp. $157 \cdot 189 ; 217 \cdot 21 ; 241 \cdot 246$. 
ces mines de fer, il y en a assez en France pour en fournir pour tout le Canada ${ }^{31}$. Deux autres faibles tentatives furent faites en 1722 et en $1724^{31}$.

Ce ne fut qu'en 1729 qu'une entreprise de forge fut lancée et soutenue. L'entrepreneur fut François Poulin de Francheville, un marchand de Montréal, et seigneur de Saint-Maurice ${ }^{32}$. Il déclara au ministre que sur sa seigneurie de Saint-Maurice il y avait des mines. L'exploitation de ces mines se ferait à ses propres frais; tout ce qu'il demandait était un monopole de vingt ans sur les mines qui pourraient se trouver de Yamachiche au Cap-de-la-Madeleine ${ }^{33}$. Il demanda aussi que lui soit donné un monopole exclusif sur la fabrication du fer. Il promettait d'ouvrir les forges deux ans après qu'il aurait reçu la concession ${ }^{34}$.

Beauharnois et Hocquart, respectivement gouverneur et intendant de la colonie, demandèrent que de Francheville jouisse du privilège. Tous deux étaient d'accord que le roi ne risquait aucun de ses deniers ${ }^{35}$, prédiction pour le moins étrange.

Le brevet du roi fut accordé à de Francheville, à Versailles, le 25 mars 1730. Il est dit, dans ce brevet, que les forges pourront être utiles pour une industrie de construction navale et pour d'autres travaux. Le monopole demandé, c'est-à-dire pour vingt ans, et le droit d'exploiter les terres de la seigneurie d'Yamachiche jusqu'aux terres de-la-Madeleine incluses, lui étaient accordés. Si de Francheville fouillait des terres déjà en culture, il devrait rembourser leurs propriétaires, mais les terres dans les seigneuries autres que celles qui lui appartenaient, pourraient être exploitées sans remboursement. Les mines et les forges devraient être en opération dans un délai de deux ans à compter de la date de l'enregistrement du brevet $^{36}$.

31. «Canada, Mines de Fer, (circa I717) \$, A.P.C., C II A, III.2, pp. $149 \cdot 150$.

32. « Vaudreuil au Conseil de la Marine, Québec, 24 octobre I722\$; \& Conseil de la Marine à Ressons, Versailles, i i avril 1724 D, A.P.C., C I I A, III'2, pp. $151^{\circ}$ $152 ;$ B, 46, p. 74 .

33. « Prançois Poulin de Francheville à Monseigneur le Comte de Maurepas, (1729) \$, A.P.C., C Ix A, pp. ror-103.

34. lbid.

35. Beauharnois et Hocquart au Ministre, Québec, 25 octobre 1729, A.P.C., C II A, 5I, p. 99.

36. \& Brevet qui permet au Sr Poulin de Francheville d'ouvrir, fouiller explojter pendant 20 ans des mines de fer en Canada, Versailles, 25 mars 1730 \. A.P.C., B, 54 , pp. $480 \cdot 482$. 
La même année, le 15 octobre 1730, de Francheville remercia le ministre pour la concession, et demanda que deux ouvriers de France obtiennent leur passage gratuit sur un navire du roi ${ }^{37}$.

Deux ans plus tard, de Francheville écrit au ministre qui n'avait rien épargné pour le développement des mines, qu'il a amené des ouvriers de France (il avait oublié que c'était aux dépens de l'État), qu'il avait même envoyé un observateur en Nouvelle-Angleterre et qu'il demandait un prêt de 10,000 livres de la caisse du Trésor de la marine au Canada ${ }^{38}$. Dans cette demande, de Francheville est soutenu par le gouverneur et l'intendant. Ces derniers soulignent que de Francheville a 30,000 livres de biens à Montréal ${ }^{30}$. Le ministre acquiesce à ces demandes et, en 1733, de Francheville béné' ficie d'une avance ou d'un prêt de 10,000 livres ${ }^{40}$.

Le 16 janvier 1733, de Francheville avait formé une compagnie pour l'exploitation des forges. Parmi les membres de cette compagnie, à part de Francheville, il y avait son frère, Pierre Poulin, François-Etienne Cugnet, Ignace Gamelin et Bricault de Valmur, le secrétaire de l'intendant Gilles Hocquart ${ }^{41}$. Cette société ne durera que jusqu'à la fin de 1733, étant donné que de Francheville est mort au mois de novembre ${ }^{42}$. Gamelin et Cugnet avaient chacun un cinquième de la compagnie. Plus tard, ils diront que leur intérêt, en termes de monnaie, était limité à 3,000 ou 4,000 livres ${ }^{43}$.

La veuve de Poulin de Francheville, Thérèse de Couagne continua l'entreprise de son mari.. Ses associés étaient les mêmes que ceux de son mari. La mort de de Francheville, et la rentrée dans l'association de Cugnet, paraissent avoir modifé la conception de

37. « Francheville au Ministre, Québec, 15 octobre I730», A.P.C., C II A, I10.2, pp. $155^{-156 .}$

38. « Prancheville à Monseigneur le Comte de Maurepas... (1732) \$; « Francheville à Maurepas, (24 octobre?) I732》, A.P.C., C II A, I12.2, pp. I51-152; 57 , pp. $205 \cdot 206$.

39. Beauharnois et Hocquart au Ministre, Québec, 15 octobre 1732 D. A.P.C., C II A, 57, pp. $200 \cdot 204$.

40. 《Ministre à Francheville, Versailles, 2 I avril $1733 \$$; Ministre à Beauhar nois et Hocquart, Versailles, 2 I avril I 733 \$ «Obligation du Sieur Francheville de roooo livres, Québec, I I octobre I733», A.P.C., B, 59.1, p. 189; I7I; C I1 A, I 10.2, pp. $177^{-1} 78$.

4I. Cession de la Veuve Francheville et du Sr Poulin du privilege de lexploitation des mines de fer, Québec, I5 octobre I736», A.P.C., C II A, IIorr, p. $28 \mathrm{r}$. 42. «Mémoire de Gamelin, 174I \$; «Obligation de la Veuve Francheville, Montréal, r9 décembre I 733 \$, A.P.C., C I I A, Irx-1, pp. 293-301; 110.1, pp. 179-181.

43. « Memoire de Demission de Cugnet, 174I \$; 《Mémoire de Gamelin, I741 》, A.P.C., C II A, II0-1, pp. 60.61 ; III. 
l'entreprise. Entre le 16 janvier 1733 et la cession au roi, par la veuve de Francheville, du privilège sur les mines, le 23 octobre 1735, les associés avaient déboursé 21,583 livres un sol et six deniers ${ }^{44}$.

La documentation ne fournit aucune explication à la Cession qu'ont fait, en 1735, la veuve de Francheville et le sieur Poulin de leur privilège ${ }^{45}$. Il est fort probable que Hocquart, sous la prese sion de Cugnet, a joué un rôle dans cette cession car Maurepas, le ministre de la Marine, écrit à un fonctionnaire du ministère, le 11 janvier 1735, qu'il a reçu demande d'envoyer deux maîtres de forge en Nouvelle-France. Il dit à Tassin que Sa Majesté a consenti et il lui demande de trouver deux hommes dont le transport en Nouvelle-France sera payé par l'Etat ${ }^{46}$.

Entre le 16 et le 25 mars de la même année, Maurepas écrit de nouveau à Tassin lui disant qu'il a reçu sa lettre. Il demande à Tassin de lui écrire les conditions auxquelles Olivier de Vézain est prêt à aller en Nouvelle-France ${ }^{47}$. Au mois de mai de la même année, le ministre de la Marine écrit aux hauts fonctionnaires de la colonie qu'il a trouvé un maitre de forge capable de diriger un établissement. Vézain a décidé d'aller en Nouvelle-France. On lui accorde une gratification de 1,200 livres pour son voyage et le salaire de 2,400 livres par année pour six ans. Le ministre dit que ces exigences sont fortes, mais que personne d'autre n'était disposé à aller dans la colonie ${ }^{48}$. L'arrivée d'Olivier de Vézain à Québec, le 7 octobre 1735, marque le commencement de la grande entreprise des forges du Saint-Maurice ${ }^{40}$. Bien que les administrateurs de la métropole ne soient pas encore prêts à se prononcer sur l'entreprise, l'intendant de la colonie, Hocquart, met les choses en marche. Il envoie Lanoullier de Boisclerc et Vézain aux environs de Trois Rivières dans le but de faire un rapport sur les mines, l'emplace. ment présent des forges et les moyens à prendre pour leur développement ${ }^{\text {so }}$.

44. \&Beauharnois et Hocquart au Ministre, Québec, 28 septembre I 734 》, A.P.C., C II A, x10.2, p. 169 .

45. «xtrait des Registres du Conseil d'État, I 743 》, A.P.C., C I I A, I IorI, pp. $34 \cdot 35$.

46. 《Maurepas à Tassin, I janvier I 735 \$, A.P.C., B, 62, pp. 8.9.

47. « Maurepas à Tassin, (entre le 16 et 25 mars) I $735 \$$, A.P.C., B, 62, p. $4 \mathrm{I}$.

48. Ministre à Beauharnois et Hocquart, Versailles, ro may I $735 \gg$, A.P.C., B, $63 \cdot 1$, pp. $237 \cdot 238$.

49. \& Ministre à Tassin, 27 décembre 1735 \$, A.P.C., B, 62, pp. xII-1 3 .

50. « Boisclerc au Ministre, Québec, 31 octobre 1735 \$ « Hocquart au Ministre, I novembre I $735 \gg$, A.P.C., C II A, 64, pp. 264.265; $247 \cdot 248$. 
Après le retour de Vézain à Québec, les deux anciens membres. de la compagnie de Francheville, Cugnet et Gamelin, et Olivier de Vézain présentent des soumissions ${ }^{51}$. La même année, un projet de dépense pour l'établissement et l'exploitation des forges est envoyé en France. Le coût total de l'établissement, d'après Vézain, sera d'environ 36,000 livres. Toujours d'après la même source, les dépenses annuelles d'exploitation s'élèveraient à 61,000 livres. Le profit anticipé est d'environ 55,000 livres par année ${ }^{52}$.

Les intéressés demandent une avance de 100,000 livres pour aider l'entreprise. Ils les obtiennent ${ }^{53}$. Si le projet avait pu être établi sur la base des soumissions, les entrepreneurs n'auraient pas eu à y investir leurs fonds propres.

L'année suivante, en 1736, un autre maitre de forge, Jacques Simonet, arriva en Nouvelle-France. Le 16 octobre de la même année, une société fut formée et l'année suivante, en 1737, un « Traité des Sociétés des forges de St-Maurice » fut établi ${ }^{54}$.

Sans entrer dans les détails, notons les noms des associés et leur fonction. Cugnet, comme on l'a mentionné précédemment, était le premier conseiller du Conseil supérieur de Québec et le directeur du Domaine d'Occident au Canada. Thomas-Jacques Taschereau, aussi conseiller au Conseil supérieur, était de plus le trésorier de la Marine en Nouvelle-France. C'est donc dire qu'il était le responsable des déboursés de l'État français en Nouvelle-France. L'autre Canadien était Ignace Gamelin, un négociant de Montréal. Ce dernier était aussi intéressé à la traite des postes des pays d'en haut, et, plus particulièrement, il était associé avec La Vérendrye dans

5x. «Offres et Soumissions. par les Sieurs Cugnet, Olivier de Vezain et Gamelin de se charger de l'Établissement des Forges de Saint Maurice, (1735) \, A.P.C., C II A, II0-I, pp. 102-108.

52. \&rojet des dépenses a faire pour l'établissement et exploitation des forges de fer an Canada, (1735) \; « Dépenses annuelles de l'exploitation, (r735) ; « Pro jet des sommes qu'il faut pour faire sept cent milliers de fer et batir la petite forgette en question, suivant le détail cy après, (I735) \, A.P.C., C Ir A, tro-1, pp. 324 $334 ; 335 \cdot 339 ; 340 \cdot 344$.

53. \& Ministre à Beauharnois et Hocquart, Versailles, 14 mars 1736 \. A.P.C., C II A, IIO.I, pp. 2I2:213.

54. \& Société entre les intéressés en l'établissement des forges des Saint Maurice, 16 octobre 1736 \$; 《raitté de Société dés forges de Saint Maurice, i I février I 737 \$, A.P.C., C I I A, rro-r, pp. $241 \cdot 25$ I ; 252.32I. Il n'est pas dans nos intentions de décrire ici tous les détails des sociétés, leur création, leurs clauses, mais plutôt de donner un aperçu général. La documentation réunie soit en notes, soit dans le texte, formera la base d'une étude plus approfondie et qui paraîtra en 1968 ou 1969. 
l'exploitation des postes de la Mer de l'Ouest. Les deux autres asso ciés, Français tous deux, étaient Vézain et Jacques Simonet. Ils étaient maîtres de forge. Il faut aussi souligner que d'après le deuxième article du traité de la société, l'intendant avait pris des intérêts dans la société. Les résultats de ce mélange d'intérêts ne doivent pas nous surprendre, étant donné que les administrateurs de la colonie et les entrepreneurs de la société étaient les mêmes.

Le grand bailleur de fonds dans l'entreprise était l'État français. La société formée en 1737 assuma l'obligation de 10,000 livres consentie par de Francheville et, plus tard, sa veuve; de plus, le roi a fourni 100,000 livres à la nouvelle compagnie formée en 1736. Le 18 octobre 1739, Hocquart, sans attendre la permission de Maurepas, fit une avance supplémentaire de 82,642 livres 7 sols et 6 deniers. Contre ces avances, les intéressés signèrent des obligations pour un total de 192,642 livres 7 sols et 6 deniers ${ }^{55}$.

Il faut ajouter à ces obligations formelles de l'Etat, la somme de 64,302 livres que Cugnet avait empruntée de la caisse du Do maine d'Occident, dont il était le directeur. Il devait à la même caisse 5,704 livres 18 sols pour de la farine qui lui avait été fournie. Comme conséquence des avances d'argent et de marchandises de commerçants de France et de la Nouvelle-France, Cugnet devait 71,035 livres 12 sols et cinq deniers, et 7,349 livres 17 sols et deux deniers à divers ouvriers et détenteurs de billets dans la colonie ${ }^{50}$. Le total des dettes de Cugnet et de ses associés était de 341,034 livres 15 sols et un denier. D'après Hocquart, les dépenses totales de la société se chiffraient à 352,642 livres 7 sols 6 deniers ${ }^{57}$. La part fournie par Cugnet s'élève alors à 11,608 livres 2 sols et 5 deniers ${ }^{58}$. La compagnie fit banqueroute en 1742 . Cugnet clama tout haut sa ruine, mais, comme nous le verrons, l'intendant Hocquart et l'Etat français agiront comme anges gardiens.

55. Compte sommaire de Balance des Dépenses faites par le Sr Cugnet... depuy le 7 may 1735 jusqu'au premier octobre 1741 , Québec, 3 octobre $174 \times \gg$, A.P.C.,

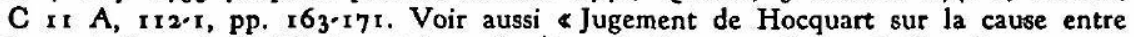
le controlleur de la Marine et les créanciers de Cugnet, 16 et 28 décembre $1741 \$$, Collection, no 1285 .

56. Memoire de Cugnet, 1742 \$, A.P.C., C II A, I 10-1, pp. 3.4.

57. « Hocquart au Ministre, Québec, 25 octobre 1742》, A.P.C., C I I A, I12.2, pp. $22 \cdot 38$.

58. Les statistiques précises sur cette entreprise sont à établir. Les données dont on se sert sont des conclusions temporaires. 


\section{L'ACTUALITE ECONOMIQUE}

En avocat habile qu'il était, Cugnet tentait une séparation de biens avec sa femme pour déjouer ses créanciers ${ }^{59}$. N'y arrivant pas, il se tourna vers l'intendant. Ce dernier, par l'entremise du contrôleur de la marine, Victor Varin de la Marre, donna un sursis à Cugnet disant que la saisie de ses meubles pourrait nuire aux droits prioritaires du roi, ce qui était d'ailleurs vrai ${ }^{60}$. Cependant, les dettes des intéressés dans les forges de Saint-Maurice demeure' ront la responsabilité de la société ${ }^{61}$. L'année suivante, soit en 1742 , Maurepas donna son approbation à la politique de Hocquart. Le ministre écrivit que les intérêts de tous devaient être protégés et qu'il laissait à Hocquart les meilleurs moyens d'y parvenir ${ }^{\mathbf{6 2}}$.

On constate que Cugnet, en dépit du fait qu'il eut emprunté une somme de la caisse du Domaine d'Occident, retiendra son poste lequel lui procure un revenu de 3,000 livres par année. Le bureau du contrôleur, dont le loyer est payé par l'Etat, se trouvait dans la maison de Cugnet. Celui-ci a gardé son poste comme premier conseiller bien qu'il fût en banqueroute ${ }^{63}$. Le bail qu'on lui avait accordé pour le poste de Tadoussac devait se terminer en 1746. Hocquart continua le bail au même prix soit, 4,500 livres par année ${ }^{64}$. Cette extension du bail était un privilège si l'on peut en croire le gouverneur Beauharnois. Ce dernier écrit au ministre que Lafontaine avait offert 10,000 livres par année, comme prix du bail en $1740^{\circ 5}$. Même si ce dernier chiffre est exagéré, on ne peut douter que Cugnet l'ait eu à bon prix, étant donné qu'en 1749, le

59. \& Jayat, Monnier, Payes, Jehanne et Bedout au Lieutenant Général Civil et Criminel au siège de la prévôte de Québec, 4 et 5 décembre I 741 , Collection, no 7247.

6o. \& Le Controlleur de la Marine à Monsieur l'Intendant de la Nouvelle-France, 14 décembre $x 74 \mathrm{I} \gg$, Collection, no $\mathrm{x} 247$. Voir aussi \&Ordonnance qui surçeoit la vente des meubles et effets de $\mathrm{Mr}$ Cugnet, 16 octobre I74r $\gg, A . P . C ., \mathrm{C} \cdot \mathrm{I}$ I A, I I0.2, pp. 24 r.243.

6I. \& Ordonnances qui, sur la démission, cession et abandon faits par les intéressés des Forges de Saint-Maurice, portes que les dites forges et effets en dépendants généralement quelconques seront et demeureront aux risques, périls et fortune des sieurs Cugnet, Gamelin, Taschereau, Olivier et Simmonet, intéressés, jusqu'a ce qu'il ait plus à Sa Majesté de donner des ordres sur les. dites démissions, cession et abandon, I 5 octobre I74I $\gg$, Roy, Ordonnances des intendants, vol. 3, pp. I 5-16.

62. « Ministre à Hocquart, Fontainebleau, I 4 may $1742 》 ;$; Ministre à Hocquart, Versailles, 25 may $174^{2} \gg$, A.P.C., B, 74'2, pp. $449 \cdot 450$; $45^{1 \cdot 454}$.

63. \& Monsieur Cugnet sur les Forges de Saint-Maurice... I7 octobre 174I», A.P.C., C I I A, I ro- I, p. 235.

64. \& Cugnet au Ministre, Québec, 23 octobre 1744 \$, A.P.C., I 1222, p. 327. p. 93 .

65. « Beauharnois au Ministre, Québec, 20 octobre 174I \$, A.P.C., C I I A, 74, 
bail de Tadoussac a été remis à Marie-Anne Barbel, veuve Fornel, contre un loyer de 7,000 livres par année ${ }^{86}$. Cugnet, comme d'habitude, prétendait qu'il perdait toujours de l'argent. Mais étant donné qu'il tenait à garder ce bail, on peut se demander s'il disait la vérité $^{87}$. Même l'ange gardien de Cugnet, c'est-à-dire l'intendant Hocquart, soutenait que le profit à Tadoussac se montait à 6,000 ou 7,000 livres par année ${ }^{68}$.

Cugnet s'est aussi vu assigner quatre postes dans les pays d'en haut, soit Népigon, Kamanistigoyan, Michipicoton et Témiscamingue. Cugnet n'exploita pas ses postes lui-même mais sous-loua plutôt son privilège à d'autres. Pour Témiscamingue, il reçut 5,600 livres par année. Michipicoton lui rapportait 3,750 livres ${ }^{69}$. Le droit d'ex' ploitation de ses quatre postes ne coûtait rien à Cugnet.

Le $1^{\mathrm{er}}$ mai 1743 , le roi révoquait le privilège accordé aux associés des forges. Les dettes des associés, et en particulier celles de Cugnet, étaient annulées en retour de leur démission ${ }^{70}$. Cugnet était alors responsable de ses dettes envers les particuliers. Ceux'ci n'ont jamais été payés, en tout cas pas avant sa mort.

Les affaires de Cugnet s'arrangèrent tant bien que mal jusqu'au départ de Hocquart et à l'arrivée de Bigot comme intendant de la Nouvelle-Prance. Ce dernier, si on lit bien la teneur de ses lettres, n’aimait pas Cugnet. Bigot accusa Cugnet de déjouer ses créanciers, et de mal gérer le Domaine d'Occident. Il passa le bail de Tadoussac à la veuve Fornel ${ }^{71}$.

Avec le départ de Hocquart et l'entrée de Bigot sur la scène, on entend peu parler de l'affaire Cugnet. En 1749, madame Bégon écrit : « on assure le rétablissement de la santé de $\mathrm{M}$. Cugnet qui a été à l'extrémité ». La même dame écrit dans son journal en date

66. «Bail de la Ferme de Tadoussac, 9 septembre r749», A.P.C., B, 94.2, f 8.

67. \& Memoire de Cugnet, Québec, 2 juillet $1740 \gg$, A.P.C., I21/2, pp. I03/1 3 r.

68. \& Hocquart au Ministre, Québec, 23 octobre 1743 ». A.P.C., C I I A, 80, p. 9x.

69. «Cugnet à Maurepas, Québec, 20 octobre 1747 》, A.P.C., E, carton 101, pp. $33.4 \mathrm{r}$. Les loyers des autres postes ne sont pas donnés.

7o. \& Arrest qui revoque le Privilege accordé aux Srs Cugnet, Gamelin, Tachereau, Olivier de Vezain et Simonet pour l'exploitation des mines de fer de St Maurice et reunit au Domaine l'Etablissement fait dans cet endroit ainsi que les effets qui en dependent, Versailles, I may $1743 \gg$, A.P.C., B, 76-1, pp. $255^{-26}$ r.

71. « Bigot au Ministre, Québec, in octobre I749 ; \& Bigot au Ministre, Québec, 29 septembre 1749 \$, A.P.C., C II A, 93, pp. 305.312; 259.260. 
du 22 décembre 1750 que Cugnet ne se porte pas bien ${ }^{72}$. Le 19 août 1751, François-Etienne Cugnet meurt ${ }^{73}$. Le jour suivant, on trouve à l'entrée de ses funérailles : «1751. 20 août. François Etienne Cugnet, premier conseiller au Conseil supérieur et directeur du domaine d'Occident, 63 ans. ${ }^{74}$. Son amie, madame Bégon, se dit très affligée. L'année suivante, en 1752, elle est plus optimiste : « on me montre que les affaires de madame Cugnet s'arrangent bien et qu'elle aura de quoi vivre à son aise, ses dettes seront ou sont presque payées ${ }^{75}$. Ceci est confirmé par l'inventaire des biens fait après la mort de Cugnet. Les réclamations des particuliers se chiffrent à 114 livres quatre sols quatre deniers. Le total des biens de la maison se chiffre à 35,638 livres 12 sols et 9 deniers. $A$ ceci, il faut ajouter la valeur de la maison, environ 60,000 livres, et les propriétés de Cugnet ${ }^{70}$. Pour un homme en banqueroute ce n'était pas si mal.

Nous nous sommes servi de Cugnet comme point de départ d'une enquête, disons, rapide et sommaire, étant donné que la documentation était à notre disposition. Bien que Cugnet, comme entrepreneur, ait eu plus de capacité que d'autres citoyens de la Nouvelle-France, ce serait exagéré de croire que son cas est unique. D'autres industries, d'autres entreprises, bien que n'ayant pas l'en' vergure des forges, se sont servies des mêmes moyens. Ce sont ces moyens que nous voulons souligner. Les hommes d'affaires dans les sociétés coloniales, soit de la France, soit de l'Angleterre, faisaient face à des problèmes qui se ressemblaient. Il y avait, dans les colonies du Nord de l'Amérique, un manque de capitaux et un marché

72. « Correspondance de Madame Bégon, Née Rocbert de la Morandière, Journal, 14 mars 1749 et 22 décembre 1750 \$, Rapport de l'archiviste de la province de 2uébec, 1934.35, pp. 47, 134. (Dorénavant Bégon).

73. E.-Z. Massicotte, « La famille du légiste François-Joseph Cugnet \$, Bulletin des recherches historiques, 1915, p. 236 .

74. P.V. Charland, « Notre-Dame de Québec : Nécrologie de la crypte \$, Bulle. tin des recherches historiques, 1914, p. 214. 178.

75. « Madame Bégon à son gendre, La Rochelle, octobre 1752》, Bégon, pp. 160,

76. «Inventaire des biens de la Comm'té entre Feu Me François-Étienne Cugnet... 31 aoust et 4 octobre $1742 \$$, A.Q., Dossier Cugnet, F.E. 
relativement restreint. Ces colonies avaient aussi en commun une balance commerciale déficitaire ${ }^{77}$. L'expérience de Cugnet est un cas typique des difficultés que connaissait un entrepreneur et des moyens auxquels il avait recours pour surmonter ces difficultés. Les commerçants, les négociants, les marchands ou les entrepreneurs de la Nouvelle-France, de la Nouvelle-Angleterre ou de New-York, qui ont eu du succès, ont été ceux qui ont su tirer avantage de leur position politique et de leur position économique. Cugnet, comme on l'a vu, était un administrateur civil, dans la judicature, commerçant, propriétaire foncier et seigneur. Il était soutenu par un haut fonctionnaire, l'intendant Gilles Hocquart. Ses associés, français ou canadiens, apportaient non seulement de l'argent ou des capitaux, mais le savoir-faire ou l'influence. C'est cette combinaison d'éléments qui aboutit au succès, succès relatif certes, des forges de Saint-Maurice. L'élément important dans toute cette histoire est le rôle de l'Etat. C'est ce dernier qui apporte les fonctions politiques et les ressources financières. Le soutien de l'Etat aboutissait à des subventions de sa part. L'intérêt que l'Etat montrait pouvait in' citer les marchands de France ou de la Nouvelle-France à faire crédit soit sous forme d'argent, soit sous forme de marchandises. L'État était aussi prêt à accepter les produits des forges en retour des avances qu'il avait faites. La banqueroute de 1742, faillite d'entre' preneur, ne conduit pas à la banqueroute totale de Cugnet. Nous devons ajouter qu'elle n'a pas eu comme résultat l'abandon des forges. Tout au contraire, celles'ci devaient durer jusqu'au XIX ${ }^{\mathrm{c}}$ siècle. L'étude de l'entreprise en Nouvelle-France doit alors dépasser l'étude de la seule banqueroute. Celle-ci n'est qu'une des conditions normales d'épanouissement de sociétés sous-développées. C'est là que la réalité se trouve et non dans le conflit allégué entre des idéologies de l'ancien régime et une éthique protestante.

\author{
Cameron NISH, \\ professeur à l'Université Sir George Williams \\ et \\ directeur de recherche, \\ Centre de Recherches en Histoire économique \\ du Canada français.
}

77. Ces données sont tirées de l'ouvrage sur La bourgoisie canadienne. Voir note 24. 


\section{APPENDICE}

L'Actualité Économique, vol. 41, no 1, avril-juin 1965 :

«Délibérations des Creanciers du Sr. Cugnet, Québec, 18 août 1742. 》, pp. 149-151.

«Etat de mes Effets actyfs et Passifs, Québec, 21, 22, 26, 27 août 1742. 》, pp. 151-165.

«Ordre pour faire l'inventaire des effets du Sr. Cugnet, Québec, 27 août 1742. 》, pp. 165-166.

«Scellées et Inventaire des Effets du Sr. François Etienne Cugnet, Québec, 28, 29, 30 août et le 1 et 3 septembre 1742. », pp. 166-202.

L'Actualité Économique, vol. 41, no 2, juillet-septembre 1965 :

« Extrait des Registres du Conseil d'Estat, Versailles, le vingt cinq May mil sept cent quarante deux. », pp. 347-348.

«Arrêt qui révoque le privilege accordé aux Srs Cugnet, Gamelin, Tachereau, Olivier de Vezain et Simonet pour l'exploita' tion des mines de fer de St Maurice et reunit au domaine l'etablissement fait dans cet endroit ainsy que les effets qui en dépendent, Versailles, 1 mai 1743. », pp. 348-351.

«Hocquart au Ministre, Canada, 23 Octobre 1743. », pp. 351353.

« Memoire de Cugnet, Québec, le vingt quatre octobre 1743. », pp. 353-362.

«A M. Cugnet, A V'les le 24 Avril 1744. », p. 462.

« Ministre à Cugnet, Versailles, 12 mai 1745. 》, pp. 362-363. «Beauharnois et Hocquart au Ministre, Québec, le $188^{\text {'bre }}$ 1746. », pp. 363-364.

«Cugnet A Monseigneur le Comte de Maurepas, Ministre et Secretaire d'Etat, Canada, 1746. », pp. 364-366.

« Cugnet au Ministre, Québec, le vint octobre 1747. », pp. 366 . 367.

«A Monseigneur le Comte de Maurepas Ministre et Secretaire d'Etat, 20 octobre 1747. », pp. 367-371.

« A. M. Hocquart, A V'les le 6 mars 1748. », pp. 371-372. 
«Cugnet au Ministre, A Québec le 20 Octobre 1748. », pp. 372-373.

«Bigot au Ministre, Québec, 11 octobre 1749. », pp. 373-375.

«Bigot au Ministre, Québec, 29 Septembre 1749. », p. 376.

« Ministre à Bigot, Compiègnes, 7 juillet 1752. », pp. 376-377.

« Bigot au Ministre, Québec, 28 Octobre 1758. », pp. 377-378.

L'Actualité Economique, vol. 41, no 4, janvier'mars 1966 :

«Cession au Roy pour la securité du Sieur Francheville dù privilège de l'exploitation des mines de fer du 23 8'bre 1735. », pp. $762-675$.

«Offres et sousmissions par les Sieurs Cugnet, Olivier de Vezain et Gamelin de se charger de l'Etablissement des forges de Saint Maurice... (1735) », pp. 765-767.

«Projet de dépenses a faire pour l'établissement et exploitation des forges de fer en Canada (1735). », pp. 767-772.

«Dépenses annuelles de l'exploitation (1735).», pp. 772-774.

« Projet des sommes qu'il faut pour faire et parfaire sept cent milliers de fer et batir la petite forgette en question, suivant le détail cy après (1735). », pp. 775-776.

«Société entre les interessés en l'établissement des forges de Saint Maurice, 16 Octobre 1736. », pp. 776-780.

«Traitté de Société des forges de Saint Maurice, 1737. », pp. 780-806.

«Etat des fonds nécessaires a l'exploitation des forges de Saint Maurice (1735). », pp. 806-810.

L'Actualité Économique, vol. 42, no 1, avril-juin 1966 :

«Le Sieur Simonet père, 9 8'bre 1741. », p. 161.

«Mémoire d'Ignace Gamelin, 9 octobre 1741. », pp. 162-165. «Olivier de Vézain a Nosseigneurs, Présenté le 13 8'bre 1741. », pp. $165-166$.

«A Nosseigneurs les Gouverneur Général et Intendant de la Nouvelle france (Thomas-Jacques Taschereau), Québec, 14 octobre 1741. », pp. 166-169. 
«Cugnet sur les forges de Saint Maurice administrées par le Sieur Olivier, Québec, 17 octobre 1741. », pp. 169-197.

«Mémoire de Cugnet, Québec, 12 Septembre 1742. », pp. 197. 208.

L'Actualité Économique, vol. 42, no 2, juillet-septembre 1966 :

«Mémoire pour servir d'instruction au Sieur Estèbe notre subdélégué a Saint-Maurice, Québec, 28 Octobre 1741. », pp. 391394.

«Etat Général de la Dépense faitte pour l'Exploitation des forges de St Maurice depuis le $1^{\text {er }}$ octobre 1741 jusqu'au $1^{\text {er }}$ aoust 1742 . 》, pp. 395-422.

L'Actualité Economique, vol. 42, no 3, octobre-décembre 1966 :

« Etat Général de la Dépense faitte pour l'Exploitation des forges de St Maurice depuis le $1^{\text {er }}$ octobre 1741 jusqu'au $1^{\text {er }}$ aoust 1742. 》 (suite et fin), pp. 704724.

«Bigot au Ministre, Québec, 29 octobre 1749. », pp. 724727. 